EDITORIAL

\title{
Considering Trends in Sodium, Trans Fat, and Saturated Fat as Key Metrics of Cardiometobolic Risk Reduction
}

\author{
Samuel F. Posner, PhD; Barbara A. Bowman, PhD; Janet L. Collins, PhD
}

\begin{abstract}
Suggested citation for this article: Posner SF, Bowman BA, Collins JL. Considering Trends in Sodium, Trans Fat, and Saturated Fat as Key Metrics of Cardiometobolic Risk Reduction. Prev Chronic Dis 2014;11:140561. DOI: http://dx.doi.org/ $10.5888 / \mathrm{pcd} 11.140561$.
\end{abstract}

The 2 articles by Urban and colleagues published this week in Preventing Chronic Disease report 15-year trends in sodium, trans fat, and saturated fat, 3 food components associated with increased risk for cardiovascular disease and obesity, in frequently ordered meal items (French fries, cheeseburgers, grilled chicken sandwiches, and regular cola) from leading US national fast food chain restaurants $(1,2)$. These longitudinal findings track these 3 food components in foods that are frequently consumed by Americans. In recent surveys, almost half of Americans report eating fast food at least weekly (http://www.gallup.com/poll/163868/fast-food-major-part-diet.aspx), and similarly, nearly half report drinking soda daily (http://www.gallup.com/poll/156116/Nearly-Half-Americans-Drink-Soda-Daily.aspx). The findings by Urban et al confirm a substantial reduction in the content of trans fat and saturated fat in French fries but not in cheeseburgers or chicken sandwiches. Changes were inconsistent in sodium, saturated fat, and calories among food products, with the exception of sodas, where there was an increase in portion size. The authors conclude that, unlike the reduction observed in artificial trans fat in French fries, the content of sodium, saturated fat, and calories in the selected foods did not change much. Taken together, these findings indicate that little improvement has been made in the quality or energy density of popular fast food products and suggest the need for interventions to improve population health.

It is important to consider these findings in the larger context as public health researchers, practitioners, and policy makers develop and implement interventions to reduce intake of excessive calories, saturated fat, and artificial trans fat. Cheeseburgers, French fries, and a soda represent a quintessential part of American culture. Banter about them was central to the Saturday Night Live skit made famous by the late John Belushi. Similarly, songs made popular by performers such as Jimmy Buffett, Charlie Pride, the Gang of Four, and the Village People are all about having a cheeseburger, French fries, and a soda. These staples of the American diet are unlikely to disappear. However, central to American food choices is an unacceptably high prevalence of diet-related risk factors that compromise the health of Americans and contribute to the high costs of chronic disease. During the period examined by Urban and colleagues, the late 1990s through 2013, the US prevalence of chronic disease risk factors such as overweight, obesity, and hypertension have remained high, cardiovascular disease remains the leading cause of death, and prevalence of prediabetes and diabetes continues to increase (3). The continued popularity of fast food restaurants and continued high prevalence of diet-related risk factors remind public health researchers, practitioners, and policy makers that there is much that needs to be done.

The findings of the 2 studies by Urban and colleagues present concrete evidence on an issue that many may consider obvious: the paradigmatic meal of a cheeseburger with French fries and a soda still contains components whose consumption is associated with increased risk of adverse health outcomes. Changes in portion size can change the amount of these components consumed, as with the increasing portion size observed for regular soda, which can increase energy density.

In the past several years, increased public health efforts have sought to implement and evaluate interventions to increase access to healthy food options through farmers markets, community gardens, and other venues that make the healthier choice the easier choice whenever possible (4-6). Changes in subsidy programs and other structural changes have been implemented to remove access barriers, such as remediation of food deserts or placement of farmers markets in high-traffic areas, such as near subway stops. Many evaluation efforts have focused on measuring consumer awareness and purchasing behavior as surrogates for consumption (5-7). Few studies have evaluated changes in key outcomes, including consumption patterns, changes in risk factors, or changes in health 
outcomes. These 3 outcomes are challenging to study because they are hard to measure, the time horizon to document an effect is long, and the attribution to a specific intervention is difficult to make. Researchers, practitioners, and policy makers need to work together to develop creative methods to study these important outcomes. Evaluating effect and return on investment of these interventions is critical to identify which interventions will make a lasting difference in the health of the population. The experience with trans fat suggests that regulatory interventions, including menu labeling in restaurants and vending machines, to be implemented in 2015 (http://www.fda.gov/NewsEvents/Newsroom/PressAnnouncements/ucm423952.htm), will improve the food environment. New federal nutrition policy, including the 2015 update of the Dietary Guidelines for Americans, will help shape the environment to support healthier choices. At the same time, there is increased public concern about the healthfulness of fast food and growing support for efforts to increase access to healthy food options, particularly in schools. Taken together, expanded policy levers in concert with increased consumer awareness and demand for healthy food products will accelerate improvement in the US diet and food environment and support reductions in the population's cardiometabolic risk.

\section{Acknowledgments}

The authors have no conflict of interest to declare.

\section{Author Information}

Corresponding Author: Samuel F. Posner, PhD, Editor in Chief, Preventing Chronic Disease: Public Health Research, Practice, and Policy, 4770 Buford Hwy, MS F-80, Atlanta, GA 30341. Telephone: 770-488-6398. Email: Shp5@cdc.gov.

Author Affiliations: Barbara A. Bowman, Janet L. Collins, National Center for Chronic Disease Prevention and Health Promotion, Centers for Disease Control and Prevention, Atlanta, Georgia.

\section{References}

1. Urban LE, Roberts SB, Fierstein JL, Gary CE, Lichtenstein AH. Temporal trends in fast-food energy, sodium, saturated fat, and trans fat content, United States, 1996-2013. Prev Chronic Dis 2014;140202.

2. Urban LE, Roberts SB, Fierstein JL, Gary CE, Lichtenstein AH. Sodium, saturated fat, and transfat content per 1,000 kilocalories: temporal trends in fast-food restaurants, United States, 2000-2013. Prev Chronic Dis 2014;140335.
3. National Center for Health Statistics. Health, United States, 2013: with special feature on prescription drugs. Hyattsville (MD): National Center for Health Statistics; 2014.

4. Cole K, McNees M, Kinney K, Fisher K, Krieger JW. Increasing access to farmers markets for beneficiaries of nutrition assistance: evaluation of the farmers market access project. Prev Chronic Dis 2013;10:E168.

5. Grin BM, Gayle TL, Saravia DC, Sanders LM. Use of farmers markets by mothers of WIC recipients, Miami-Dade County, Florida, 2011. Prev Chronic Dis 2013;10:E95.

6. Li KY, Cromley EK, Fox AM, Horowitz CR. Evaluation of the placement of mobile fruit and vegetable vendors to alleviate food deserts in New York City. Prev Chronic Dis 2014; 11:E158.

7. Payne GH, Wethington H, Olsho L, Jernigan J, Farris R, Walker DK. Implementing a farmers' market incentive program: perspectives on the New York City Health Bucks Program. Prev Chronic Dis 2013;10:E145.

\footnotetext{
The opinions expressed by authors contributing to this journal do not necessarily reflect the opinions of the U.S. Department of Health and Human Services, the Public Health Service, the Centers for Disease Control and Prevention, or the authors' affiliated institutions.
} 